\title{
The Future of Psychiatry as Clinical Neuroscience
}

\author{
Charles F. Reynolds III, MD, \\ University of Pittsburgh Medical Center Endowed Professor of Geriatric Psychiatry, director, \\ Advanced Center for Interventions and Services Research for Late-Life Mood Disorders, and \\ director, John A. Hartford Center of Excellence in Geriatric Psychiatry, Department of Psychiatry, \\ University of Pittsburgh School of Medicine, Pittsburgh, Pennsylvania.
}

\section{David A. Lewis, MD,}

University of Pittsburgh Medical Center Endowed Professor in Translational Neuroscience, Departments of Psychiatry and Neuroscience, and director, Translational Neuroscience Program and Conte Center for the Neuroscience of Mental Disorders, University of Pittsburgh School of Medicine, Pittsburgh, Pennsylvania.

\section{Thomas Detre, MD,}

Emeritus distinguished senior vice chancellor and health sciences, and emeritus distinguished service professor of psychiatry, University of Pittsburgh School of Medicine, Pittsburgh, Pennsylvania.

\author{
Alan F. Schatzberg, MD, and \\ Kenneth T. Norris, Jr. Professor and chairman, Department of Psychiatry and Behavioral Sciences, \\ Stanford University School of Medicine, Palo Alto, California.

\section{David J. Kupfer, MD} \\ Thomas Detre Professor and chair, Department of Psychiatry, University of Pittsburgh School of \\ Medicine, Pittsburgh, Pennsylvania.
}

\begin{abstract}
Psychiatry includes the assessment, treatment, and prevention of complex brain disorders, such as depression, bipolar disorder, anxiety disorders, schizophrenia, developmental disorders (e.g., autism), and neurodegenerative disorders (e.g., Alzheimer dementia). Its core mission is to prevent and alleviate the distress and impairment caused by these disorders, which account for a substantial part of the global burden of illness-related disability. Psychiatry is grounded in clinical neuroscience. Its core mission, now and in the future, is best served within this context because advances in assessment, treatment, and prevention of brain disorders are likely to originate from studies of etiology and pathophysiology based in clinical and translational neuroscience. To ensure its broad public health relevance in the future, psychiatry must also bridge science and service, ensuring that those who need the benefits of its science are also its beneficiaries. To do so effectively, psychiatry as clinical neuroscience must strengthen its partnerships with the disciplines of public health (including epidemiology), community and behavioral health science, and health economics.
\end{abstract}

The authors present a Strengths, Weaknesses, Opportunities, and Threats (SWOT) analysis of psychiatry and identify strategies for strengthening its future and increasing its relevance to public health and the rest of medicine. These strategies encompass new approaches to strengthening the relationship between psychiatry and neurology, financing psychiatry's mission, emphasizing early and sustained multidisciplinary training (research and clinical), bolstering the academic

Correspondence should be addressed to Dr. Reynolds, University of Pittsburgh School of Medicine, Western Psychiatric Institute and Clinic, 3811 O'Hara Street, E-1135, Pittsburgh, PA 15213; telephone: (412) 246-6414; fax: (412) 246-5300; (reynoldscf@ upmc.edu). 
infrastructure, and reorganizing and refinancing mental health services both for preventive intervention and cost-effective chronic disease management.

Psychiatry is the medical specialty that seeks to help (i.e., assess and treat) people and families living with complex brain disorders including depression, bipolar disorder, anxiety disorders, schizophrenia, substance abuse disorders, developmental disorders such as autism, and neurodegenerative disorders such as Alzheimer dementia. Traditionally, disorders falling into the province of psychiatry have been those of unknown etiology, and, as researchers have ascertained etiology, some disorders, such as central nervous system disorders, have often shifted to the province of neurology. (Tertiary syphilis is a good historical example of this shift.) Now, however, with the tools of modern neuroscience, a deeper understanding of causal pathways to major neuropsychiatric illness is evolving, thus rendering artificial the boundary between psychiatry and neurology. ${ }^{1}$ The artificiality of this boundary has profound implications for psychiatry's future. Our thesis is that the two disciplines, which were once united, should be, at least partially, reintegrated as clinical neuroscience (Figure 1). That said, we also acknowledge that whereas psychiatrists and neurologists share a common interest in the central nervous system, their interests can and often do diverge, resulting in group affiliations that are key for professional identity. This identity is a fundamental tenet of medical sociology. We are not suggesting that psychiatrists or neurologists give up what really interests them, but a partial reintegration of the two disciplines, especially in undergraduate and graduate medical training, as well as in research, could strengthen both. The components of psychiatry and the components of neurology are often arbitrary and historical rather than rational. Whereas neurology has traditionally focused on discrete anatomical lesions (e.g., stroke or tumors), psychiatry or modern clinical neuroscience addresses dysfunction in anatomical circuits and connectivity. These, as we illustrate below, are differences of degree-not absolutes.

According to the World Health Organization, neuropsychiatric disorders account for at least $20 \%$ of the global burden of illness-related disability, and all represent complex disorders of brain function. ${ }^{2}$ Psychiatry, like neurology, rests on a foundation of clinical neuroscience. It also encompasses and is informed by a broad range of basic biological and social sciences and has at its disposal many tools (e.g., brain imaging, genetics, neuropsychopharmacology, neurophysiology, epidemiological models of risk and protective factors, and neuropsychology) for developing new assessment and treatment approaches, grounded in understanding of etiology and pathophysiology.

Our purpose is to discuss the future of psychiatry as clinical neuroscience and to specify strategies in several contexts to enhance this future: (1) education and training, (2) health science policy, (3) institutional issues, (4) research, and (5) clinical practice in medical schools and training hospitals. We aim to present a synthesis of the key conceptual and social issues facing psychiatry right now and to provide a tool for further discussion in many areas, both in psychiatry and in academic medicine more broadly.

To explore these contexts and to provide a rationale for our views, we propose first to present a Strengths, Weaknesses, Opportunities, and Threats (SWOT) analysis of the field of psychiatry. Collectively, we bring to this task approximately 200 years of experience in all aspects of academic psychiatry: research (basic and clinical), education (including leadership of programs in mentoring for academic careers), clinical practice, and administration (medical school dean and academic health center [AHC] president, department chair, and large research group leader). All of us have significant experience with the National Institutes of Health (NIH) as reviewers, grantees, and National Institute of Mental Health (NIMH) national advisory council members; with the Institute of Medicine (IOM); and as leaders of professional associations (American Psychiatric Association [APA], the American College of Neuropsychopharmacology, and the American College of Psychiatrists). We emphasize that 
the views presented here are our own and do not necessarily reflect the official policies of any professional organization with which we are affiliated. Our purpose is not to conduct a literature review and analysis of the conceptual and social issues facing psychiatry but, rather, to synthesize a broad-based view of these issues, to broaden participation of academic medicine leaders in this conversation, and, above all, to make what we feel are strategic recommendations for dealing with the tipping point where psychiatry now balances. We think, in short, that there is an urgency to sustaining this conversation and acting on it. We note several resources in the literature of considerable value in this context from the surgeon general, ${ }^{3}$ the IOM,${ }^{4}$ and the APA. ${ }^{5}$

\section{Current Strengths of Psychiatry}

In our opinion, the assessment tools of psychiatry (e.g., the use of structured diagnostic instruments) and its treatment armamentarium (including maintenance pharmacotherapy to prevent relapse and recurrence) are good, but they are far from excellent. Patients often improve substantially, but many do not recover fully. The field has taken a largely descriptive and categorical approach to diagnosis, and now experts and practitioners recognize the need to incorporate multiple dimensions (e.g., severity, distress, impairment) into their assessment procedures to better accommodate advances in relevant basic brain and behavioral sciences and to enhance clinical relevance. Indeed, this multidimensional assessment is a fundamental goal of the Diagnostic and Statistical Manual (fifth edition) task force.

Another strength is the systematic evidence base now available to inform psychiatric treatment, which is of a relatively high order similar to, if not better than, the rest of medicine in terms of rigorously controlled randomized clinical trials (RCTs), including those testing theoretically based, disorder-specific psychosocial treatments (e.g., cognitive behavior therapy for depression). Third, as highlighted by the recent NIMH Strategic Plan, the field is scientifically committed to both optimizing treatment outcomes and personalizing treatment for those living with psychiatric disorders. ${ }^{6}$ This commitment increasingly has inspired investigating moderators of treatment responses and creating models of care organization (e.g., depression care management) that allow evidence-based practice to reach both specialty mental health and general medicine settings. These developments have also helped to foster a growing emphasis on stepped-care approaches with public health relevance (e.g., the use of watchful waiting before intervention and the use of simple interventions before more complex strategies) as well as integrated multicomponent interventions that take into account the burdens of coexisting medical, neurological, and psychosocial challenges (e.g., care-giving burden). Such interventions especially emphasize health-related quality of life. Selective and indicated preventive intervention for depression is also beginning to emerge as a feasible and effective strategy in primary care and specialty settings; such strategies recruit individuals at high risk for psychiatric illness, many of whom are already experiencing presyndromal symptoms. ${ }^{7}$ Some preventive interventions are psychosocial, such as the use of problem-solving therapy to prevent or delay depression in people living with macular degeneration ${ }^{8}$; others are psychopharmacologic, such as antidepressant medications for poststroke patients at risk for depression. ${ }^{9}$

In addition, one of the greatest strengths of psychiatry is the cadre of young people entering the field and its related basic and applied disciplines. Slightly more than $4 \%$ of graduating seniors in the nation's medical schools enter psychiatry. ${ }^{10}$

Finally, a critical and growing strength of psychiatry as clinical neuroscience derives from the multiple and marked advances that are transpiring in the areas of molecular, developmental, and systems neuroscience. The expanding knowledge of the neural substrates for the cognitive and affective functions that are disturbed in psychiatric disorders is creating an increasingly 
more sophisticated and nuanced database for the generation of testable hypotheses about the biological underpinnings of psychiatric illness. Consistent with these advances, clinical neuroscientists are now witnessing the early phases of the development of novel pharmacological interventions centered on pathophysiologically based illness models rather than on serendipitous discoveries. Psychiatrists are also now better able to estimate risk for mental illness based on genetic information and to predict treatment response variability using pharmacogenetic information. In this sense, the traditional boundaries between psychiatry and neurology, between mind and brain, are disappearing. ${ }^{11}$

\section{Current Weaknesses of Psychiatry}

Psychiatry's assessment and treatment tools, though good, are limited by the lack of - and often internal resistance to- the clinical neuroscience perspectives needed to bring the findings of psychiatric genetics, brain imaging, cognitive and affective neuroscience, and psychometric theory to defining etiology, pathophysiology, and treatment-relevant phenotypes, and to personalizing treatment (i.e., which treatment for which patient at what point in the illness trajectory?). In our opinion, optimizing treatment entails not only RCTs but also a greater emphasis on the identification of biological and psychosocial variables that predict or modify short- and long-term treatment response. As a field, psychiatry is relatively new to this enterprise, well behind many other medical specialties. We believe that the disciplinary separation of the two major practice arms of clinical neuroscience, psychiatry and neurology, is a conceptual and structural impediment to scientific and clinical progress in the care of people living with complex brain disorders. As a discipline of clinical neuroscience, psychiatry needs to invest greater scientific effort into studies of the etiology and pathophysiology of major brain disorders and to ensure that advances in these two fields are integrated appropriately into undergraduate and graduate medical education. Psychiatry has other weaknesses, too; it has paid too little attention to

- inequalities in the delivery of mental health services to vulnerable populations;

- the integration of mental health services into other areas of medicine, from pediatrics to geriatrics;

- the real and perceived conflicts of interest in relationships with industry; and

- the unmet mental health needs of medical students and physicians generally (whose rates of suicide are two to three times greater than in the general population ${ }^{12}$ ).

Finally, relative to most other specialties in academic medicine, the number of researchintensive departments of psychiatry is relatively small, probably not more the $20 \%$ of the nation's medical schools, as judged by the geographic distribution of NIH-sponsored research. ${ }^{6}$ This reflects a nexus of several challenges to the field: (1) too few psychiatrists who have completed research fellowships, (2) too few mentors, (3) an overly rigid approach to graduate medical education with inadequate flexibility to allow the integration of research training into subspecialty clinical training, and (4) a failure to recruit a fair share of the best and brightest medical students early enough into clinical neuroscience research. ${ }^{13}$

\section{Current Opportunities for Psychiatry}

Embedded within the strengths and weaknesses of psychiatry as a discipline of clinical neuroscience are tremendous opportunities to conduct research into the causes of mental illness; to chart the developmental trajectories of mental illness so as to determine when, where, and how to intervene; to develop mental health treatments and approaches responsive to diverse needs and circumstances; and to strengthen the impact of treatments for mental illnesses on public health. The tools of psychiatry's basic and behavioral sciences now permit such progress. In addition, the field is ripe with other opportunities to develop selective and indicated 
preventive interventions for people at high risk for mental illness across the lifecycle, thereby enhancing the public health impact of modern psychiatric treatment. Psychiatry can also improve both assessment and treatment strategies via deeper understanding of genetics, pathophysiology, functional neuroanatomy, and neuropsychopharmacology, allowing for the development of more personalized interventions. The opportunity to develop and implement organization models of mental health service delivery that have public health relevance will further psychiatry's reach and allow it to combat stigma against the mentally ill. The pursuit of advocacy and consumer health information initiatives through partnerships with patients and families living with mental illness is extraordinarily important to the campaign to improve payment of mental health services via parity and, in a related vein, to improve financial incentives for young people to enter the field. Finally, psychiatry has a duty to change the institutional culture of academic medicine in a way that supports medical students and physicians seeking mental health services for themselves. For example, teaching medical students and physicians to better recognize depression in themselves and in their colleagues may lead to decreased rates of physician disability and suicide—and increase the likelihood that nonpsychiatrist physicians will recognize depression in their own patients. ${ }^{12}$

\section{Current Threats to Psychiatry}

Notwithstanding the many opportunities available to psychiatry as clinical neuroscience, the field also faces challenges that threaten its future. Some of these are financial in nature (e.g., lack of parity in reimbursement policies and Medicare's discriminatory copayment requirement of 50\%). Another relates to the organization of medical practice; structural barriers (e.g., the lack of electronic health records, decreasing institutional support, and orientation to acute rather than chronic care) impede the implementation of evidence-based mental health services in general medicine and pediatric practices. Social realities, such as the persistence of stigma against the mentally ill, and health/science policy (e.g., the inadequate funding of mental health research and the scanty support for mentoring), also threaten the future of psychiatry. The dearth of recruitment of excellent students and the attendant workforce implications (e.g., the paucity of child/adolescent and geriatric psychiatrists, the low number of psychiatric trainees embarking on research careers) also loom as threats to psychiatry's future. Finally, the infrastructure needs of psychiatric and mental health research-from basic laboratories to community-based partnerships, especially with disadvantaged, underserved people-require ongoing, planned investment. The capital available for that investment is shrinking (the purchasing power of the NIMH/NIH appropriations has decreased dramatically in the past five years) and underscores the vital role of the nation's philanthropic community and appropriate partnerships with industry to ensure a robust future for psychiatry as clinical neuroscience.

\section{Strategies to Ensure the Future of Psychiatry as Clinical Neuroscience}

On the basis of this SWOT analysis, we think that the nation's health policy leaders, AHC and academic psychiatry department leaders, and important gatekeepers such as the Liaison Committee for Medical Education and the Residency Review Committees should consider the strategies listed below. We organize these strategies into the domains of (1) education and training, (2) health science policy, (3) institutional issues, (4) research practice, and (5) clinical practice.

\section{Education and training}

Education, training, and mentoring are obviously critical to ensuring recruitment and retention; therefore, we recommend the following strategies: 
- Introduce medical students to the excitement of clinical neuroscience in psychiatry during the first year of medical school. Basic neuroscience, psychiatry, and neurology faculty should coteach these courses, integrating perspectives across their disciplines.

- Recruit medical students into summer research electives in psychiatry and clinical neuroscience by providing mentoring and shadowing opportunities. Integrate early clinical exposure to patients living with complex brain disorders into these experiences to influence medical student choice for psychiatry (and neurology) in graduate medical education.

- Integrate the third-year medical school clerkships in psychiatry and neurology rather than having two separate clerkships.

- Teach medical students how to recognize depression in themselves and in their colleagues; encourage them to use mental health services and, thereby, combat the internal and external stigmas against mental illnesses.

- $\quad$ Train psychiatric residents in general medical and pediatric settings, in addition to those in specialty mental health settings, to convey mental health expertise to their medical colleagues and patients.

- $\quad$ Provide psychiatric residents in community settings with culturally appropriate skills for dealing with patients who are disadvantaged and/or underserved.

- $\quad$ Foster greater cross-training during psychiatry and neurology residencies, particularly in central (as apposed to peripheral) nervous system disorders. Psychiatrists need to know much more about brain function, and neurologists need to know much more about the neuropsychiatric dimensions of their patients' illnesses. (There is arguably more similarity between Parkinson disease and depression or schizophrenia than between Parkinson disease and myasthemia gravis or carpal tunnel syndrome.)

- Expand the education of psychiatry residents in a disease-relevant understanding of molecular, developmental, and systems neuroscience to ensure their ability to bring future advances in these areas into clinical practice.

\section{Health/science policy}

We believe that there are several critical policy issues that demand action:

- $\quad$ Pass and enforce legislation calling for parity of coverage for physical and mental illness.

- Abolish discriminatory Medicare policies requiring 50\% copayments for mental health services.

- Enhance financial incentives for medical students to choose careers in clinical neuroscience, such as loan repayment programs and improved reimbursement schedules.

- Increase federal investment in (1) research into the causes, diagnoses, and treatments of complex brain disorders and in (2) services to people with severe and persistent mental illness and other complex brain disorders.

- Foster integrated approaches by the NIMH, Institute of Neurological Disorders and Stroke, National Institute on Drug Abuse, and National Institute on Alcohol Abuse and Alcoholism to complement the federal sponsorship of research in clinical neuroscience. 


\section{Institutional issues}

Several key issues require the attention of institutional leadership. We recommend the following strategies:

- Work toward the reintegration of undergraduate and graduate medical education in psychiatry and neurology under the rubric of clinical neuroscience; offer certification by the American Board of Psychiatry and Neurology in clinical neuroscience with subspecialty qualification in psychiatry or neurology to recognize the divergent interests and group affiliations that are key for professional identity.

- Create endowments for both junior and senior professors of clinical neuroscience so as to ensure opportunities for initiating and maintaining academic career development.

- Create infrastructure to support core laboratories in clinical neuroscience and related disciplines (1) to foster future integration across these disciplines and (2) to provide interdisciplinary research and clinical training.

- Remove barriers to the use of mental health services by medical students and physicians.

\section{Research practice}

New models of research practice should include the following strategies:

- Develop broad programmatic approaches to multidisciplinary research in clinical neuroscience. These approaches should encompass (1) discovery research, (2) translational research that develops new assessment and treatment strategies informed by basic science, and (3) interventions and services research informed by ethnography and health economics.

- Institute centers of excellence in clinical neuroscience research that serve as platforms for the mentoring of pre- and postdoctoral students and of junior faculty who are involved in clinical neuroscience research.

\section{Clinical practice}

Actions to create new models of clinical practice are also much in need:

- Seek ways for those involved in the clinical practice of psychiatry in AHCs to bridge science and service both by testing models of care that facilitate implementation of evidence-based practices and by assessing their scalability in collaboration with health plans and other payors.

- Further integrate the clinical practice of psychiatry into other medical settings, particularly the primary care disciplines of pediatrics, general medicine, obstetricsgynecology, and geriatrics.

- Use non-mental-health specialty settings to teaching nonpsychiatrist physicians how to (1) screen for mental illnesses such as depression, (2) provide initial treatment, and (3) know when to refer their patients to mental health specialty care.

\section{In Sum}

As a medical discipline within clinical neuroscience, psychiatry faces many challenges. On the basis of our SWOT analysis, we believe that there are essential strategies for strengthening psychiatry's future, enhancing its public health relevance, and underscoring its importance to the rest of medicine. These strategies broadly encompass partial reintegration with neurology as a clinical neuroscience discipline, new approaches to financing its mission, emphasis on 
early and sustained multidisciplinary training, strengthening its academic infrastructure, and reengineering the organization and financing of mental health services to encourage preventive intervention and cost-effective chronic disease management integrated into, and not carved out of, the rest of medicine.

\section{Acknowledgments}

The authors were supported in part by P30 MH071944, Advanced Center for Interventions and Services Research for Late-Life Mood Disorders; NIH RR 024153 Clinical and Translational Science Institute (C.F.R., D.J.K.); and the University of Pittsburgh Medical Center endowments in geriatric psychiatry (C.F.R.); and Neuroscience (D.A.L.).

\section{References}

1. Detre T. The future of psychiatry. Am J Psychiatry 1987;144:621-625. [PubMed: 3578570]

2. Murray, CJL.; Lopez, AD. The World Health Organization and the World Bank. Global Health Statistics: A Compendium of Incidence, Prevalence and Mortality Estimates for Over 200 Conditions. Cambridge, Mass: Harvard University Press; 1996.

3. U.S. Department of Health and Human Services. Chapter 8: A vision for the future. Mental Health: A Report of the Surgeon General. [Accessed December 9, 2008]. Available at: (http://www.surgeongeneral.gov/library/mentalhealth/chapter8/sec1.html).

4. Institute of Medicine. Reports on mental health. [Accessed December 9, 2008]. Available at: (http://www.iom.edu/CMS/3704.aspx).

5. American Psychiatric Association. Task force reports. [Accessed December 9, 2008]. Available at: (http://www.psych.org/MainMenu/EducationCareerDevelopment/Library/ TaskForceReports_1.aspx).

6. National Institutes of Health, National Institute of Mental Health. The National Institute of Mental Health Strategic Plan. [Accessed December 17, 2008]. Available at: (http://www.nimh.nih.gov/about/strategic-planning-reports/index.shtml).

7. Mrazek, P.; Haggerty, R., editors. Reducing Risks for Mental Disorders: Frontiers for Preventive Intervention Research. Washington, DC: National Academy Press; 1994.

8. Rovner BW, Casten RJ, Hegel MT, Leiby BE, Tasman WS. Preventing depression in age-related macular degeneration. Arch Gen Psychiatry 2007;64:886-892. [PubMed: 17679633]

9. Robinson RG, Jorge RE, Moser DJ, et al. Escitalopram and problem-solving therapy for prevention of poststroke depression: A randomized controlled trial. JAMA 2008;299:2391-2400. [PubMed: 18505948]

10. National Resident Matching Program. Results and Data: 2008 Main Residency Match. [Accessed December 18, 2008]. Available at: (http://www.nrmp.org/data/resultsanddata2008.pdf).

11. Detre T, McDonald MC. Managed care and the future of psychiatry. Arch Gen Psychiatry 1997;54:201-204. [PubMed: 9075459]

12. Center C, Davis M, Detre T, et al. Confronting depression and suicide in physicians: A consensus statement. JAMA 2003;289:3161-3166. [PubMed: 12813122]

13. Kupfer DJ, Hyman S, Schatzberg AF, Pincus HA, Reynolds CF III. Recruiting and retaining future generations of physician scientists in mental health. Arch Gen Psychiatry 2002;59:657-660. [PubMed: 12090819] 


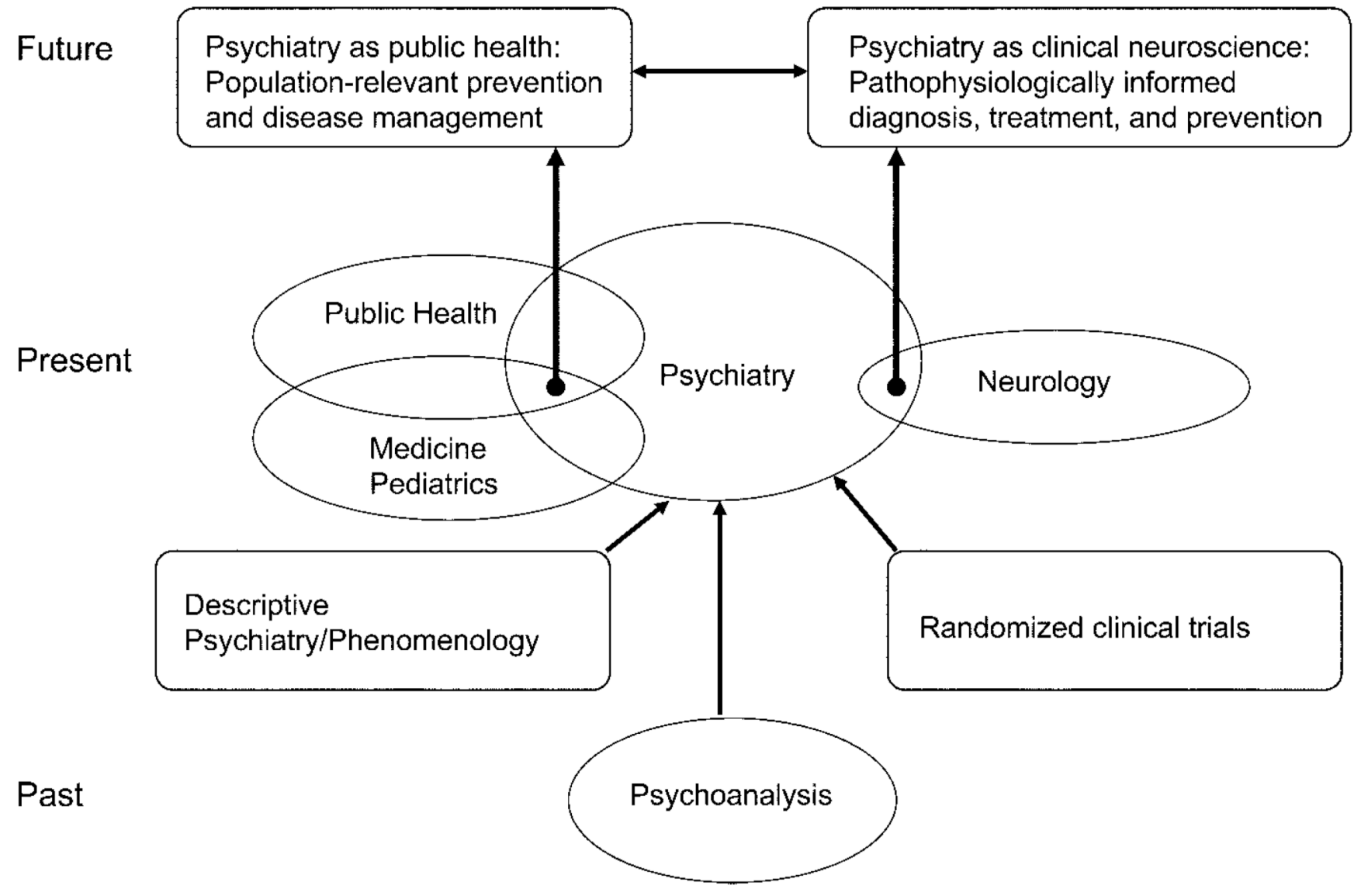

Figure 1.

The Future of Psychiatry at a Tipping Point. This concept map illustrates past streams of influence on psychiatry, its current relationship with medicine, neurology, and public health, and its future as clinical neuroscience and as a part of public-health-relevant prevention and disease management strategies. 